\begin{tabular}{|} 
Ambiente \& Água - An Interdisciplinary Journal of Applied Science \\
ISSN 1980-993X - doi:10.4136/1980-993X \\
www.ambi-agua.net \\
E-mail: ambi.agua@gmail.com
\end{tabular}

\title{
Análise multivariada para seleção de parâmetros de monitoramento em manancial de Juiz de Fora, Minas Gerais
}

\author{
doi:10.4136/ambi-agua.1590
}

Received: 29 Dec. 2014; Accepted: 04 Dec. 2015

\author{
Cézar Henrique Barra Rocha*; Amanda Maia Pereira \\ Universidade Federal de Juiz de Fora (UFJF), Juiz de Fora, MG, Brasil \\ Núcleo de Análise Geo Ambiental (NAGEA) \\ *Autor correspondente: email: barra.rocha@gmail.com, \\ amanda_maiap@hotmail.com
}

\section{RESUMO}

A necessidade de controlar o uso da terra e monitorar a qualidade da água para abastecimento público requer a avaliação de diversos parâmetros, os quais muitas vezes são difíceis de analisar e interpretar devido à grande quantidade de dados, além do custo financeiro. Nesse contexto, este trabalho teve como objetivo selecionar parâmetros mais relevantes para monitoramento da Represa Dr. João Penido, principal manancial de abastecimento de Juiz de Fora, MG. Essa avaliação foi realizada em uma série mensal de treze parâmetros fornecidos pela Companhia de Saneamento Municipal de Juiz de Fora entre os anos de 1998 a 2012, Estes dados foram avaliados estatisticamente usando Análise Fatorial e Análise de Componentes Principais, resultando na obtenção de parâmetros com maior variância. A componente 1 (FV1) refletiu a influência de sólidos dissolvidos associados aos processos de escoamento superficial na sub-bacia e erosão das margens. A FV2, assim como a FV3, associaram-se fortemente a possíveis lançamentos de esgoto no corpo hídrico. Com a análise multivariada, eliminaram-se cinco parâmetros que não apresentaram variância: dureza, oxigênio consumido, $\mathrm{pH}$, alcalinidade e manganês.

Palavras-chave: AF/ACP, qualidade da água, uso da terra.

\section{Multivariate Analysis for selection of monitoring parameters in water reservoir of Juiz de Fora, Minas Gerais}

\section{ABSTRACT}

The need to control land use and monitor water quality for public supply requires the evaluation of many parameters, which are often difficult to analyze and interpret due to cost and the large volume of data. In this context, this work selected the most relevant parameters for monitoring the Dr. João Penido Dam, the main supply source of Juiz de Fora, MG. The evaluation was conducted based upon a series of thirteen parameters of the catchment provided monthly by the Municipal Sanitation Company between 1998 and 2012. The data were statistically processed using Factor Analysis and Principal Component Analysis and parameters with highest variances were extracted. Component 1 (FV1) reflected the influence of dissolved solids associated with runoff processes in the watershed and with 
the erosion of margins. FV2, as well as FV3, are strongly associated to the potential discharge of sewage in the watershed area. Using multivariate analysis, we removed five parameters that showed no variance: hardness, consumed oxygen, $\mathrm{pH}$, alkalinity and manganese.

Keywords: FA/PCA, land use, water quality.

\section{INTRODUÇÃO}

No último censo oficial, a população de Juiz de Fora foi de 516.247 habitantes com um acréscimo de 13,37\% em relação ao ano de 2000 (IBGE, 2014). Isso tem ocasionado maiores demanda hídrica e pressão sobre os mananciais destinados ao abastecimento público. Paralelamente, a especulação imobiliária avançou sobre as sub-bacias de mananciais de Juiz de Fora, destacando a Represa Dr. João Penido. Ela tem área de aproximadamente $59,48 \mathrm{~km}^{2}$ e abastece cerca de $50 \%$ da cidade com vazão regularizada de 750 litros/segundo, chegando a até $65 \%$ no período da estiagem (CESAMA, 2014). A legislação e a fiscalização não foram capazes de evitar este processo, colocando em risco o futuro desse manancial.

Machado (2006) considera a Lei Municipal $n^{0}$ 6.087 / 1981 (Juiz de Fora, 1981) um avanço na proteção da represa, contudo, ressalva que ela é baseada em critérios pouco científicos, estando mais ligada ao processo de ocupação do que na manutenção das qualidades mínimas de suas águas. Esta ponderação ficou ainda mais evidente com a alteração do artigo $9^{\circ}$ pela Lei Municipal $n^{\circ} 11.817$ / 2009 (Juiz de Fora, 2009) que possibilita a "implantação de infraestrutura de alto interesse público" em áreas consideradas de preservação. Isso foi um artifício jurídico para a construção de uma Rodovia Estadual (Rodovia do Aeroporto) que induzirá ocupação antrópica a médio e longo prazo.

A Lei Municipal no 6.910 / 1986 (Juiz de Fora, 1986) instituiu a Bacia Hidrográfica da Represa Dr. João Penido como Zona Especial, sujeita a regime urbanístico específico que visa à preservação dos seus recursos naturais e a proteção ambiental e ecológica. $\mathrm{O}$ ideal seria a preservação de grandes fragmentos de floresta em toda a área da bacia, contudo, as matas ripárias nas margens já conseguiriam muitas vantagens para o manancial envolvido. São as funções previstas para as áreas de preservação permanente (APP), depreciadas na reformulação do Novo Código Florestal (Ab'Sáber, 2010; Casatti, 2010; Tundisi e Tundisi, 2010; Brasil, 2012a; 2012b, Rocha e Costa, 2015).

$\mathrm{O}$ adequado gerenciamento dos recursos hídricos tem sido uma constante preocupação dos gestores públicos. Para que haja fornecimento de água com boa qualidade e quantidade é necessário estabelecer um planejamento do uso e cobertura da terra nas bacias de mananciais de abastecimento (Bhattacharyya e Kapil, 2010; Tu, 2011; Sheela et al., 2012; Thurston et al., 2012; Abildtrup et al., 2013; Yu et al., 2013; Rocha et al., 2014).

Diante deste contexto, verifica-se a necessidade de um planejamento para a ocupação da região em que está inserida a Represa e também um monitoramento contínuo de suas águas. Tal monitoramento visa o controle da qualidade da água e busca avaliar diversos parâmetros, os quais muitas vezes são difíceis de analisar e interpretar devido à grande quantidade de dados, principalmente se as coletas e as análises de água ocorrerem por muitos anos. A fim de suplantar esse obstáculo é feita a utilização de abordagens multivariadas como a Análise Fatorial (AF) e a Análise de Componentes Principais (ACP) que são úteis para obter informações significativas, promovendo a redução do número de variáveis com o mínimo de perdas das informações (Liao et al., 2008; Bernardi et al., 2009; Autin e Edwards, 2010; Vanzela et al., 2010; Akbar et al., 2011; Mendonça e Souza, 2011; Spezia et al., 2011; Tu, 2011; Baborowski et al., 2012; Guedes et al., 2012; Sheela et al., 2012; Rocha et al., 2014).

Com base no que foi abordado, teve-se por objetivo selecionar os parâmetros mais 
relevantes para monitoramento da Represa Dr. João Penido, principal manancial de abastecimento de Juiz de Fora, MG, verificando pela Análise Fatorial / Análise de Componentes Principais os parâmetros que melhor explicam a variância total dos dados, ou seja, os mais relevantes para esta sub-bacia.

\section{MATERIAIS E MÉTODOS}

As informações limnológicas utilizadas foram fornecidas pela CESAMA - Companhia de Saneamento Municipal de Juiz de Fora e são referentes às análises da água na captação da Represa Dr. João Penido, com coordenadas geográficas: $2^{\circ} 41^{\prime} 03,3^{\prime \prime} \mathrm{S}$ e $43^{\circ} 24^{\prime} 12,68^{\prime \prime} \mathrm{W}$ (Datum WGS-84), localizada ao norte do município, $10 \mathrm{~km}$ da malha urbana. A construção da Represa Dr. João Penido se deu com o barramento do ribeirão dos Burros (córrego principal), contando também com os córregos Grama (ao centro), Vista Alegre (a leste) e outros tributários conforme a Figura 1.
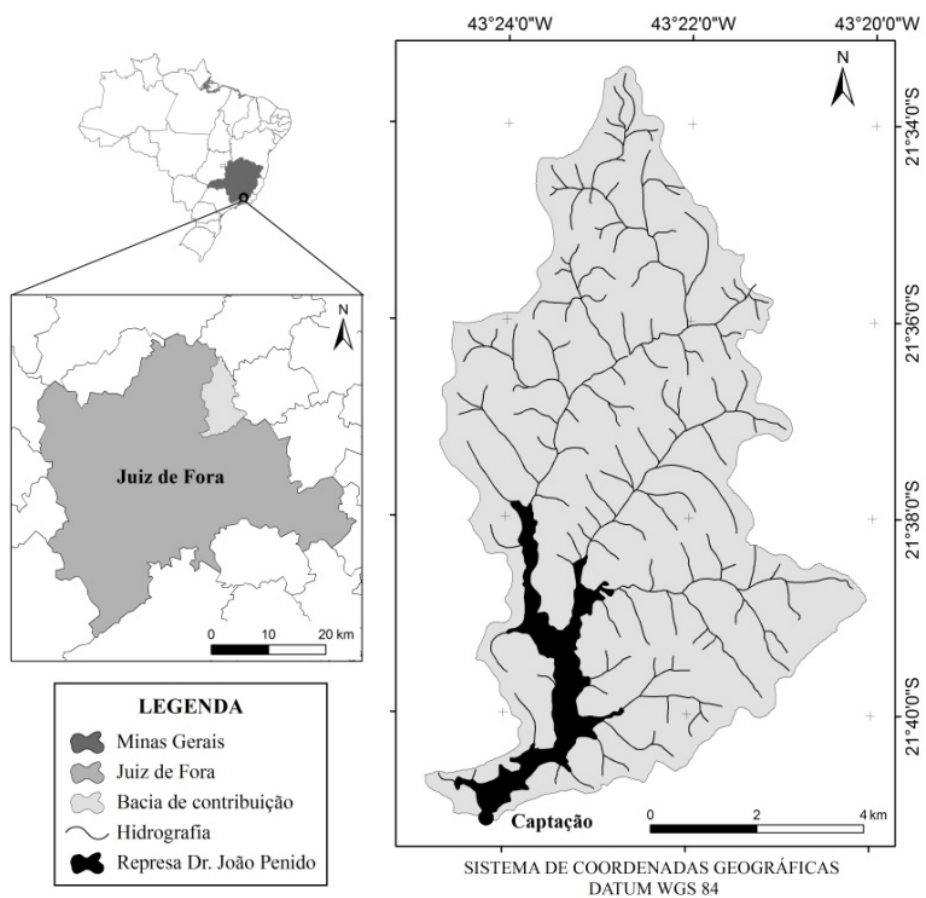

Figura 1. Mapa de Localização da área de estudo.

Fonte: Rocha et al. (2014).

Foram analisados os seguintes parâmetros: $\mathrm{pH}$, cor, turbidez, ferro (Fe), manganês (Mn), fósforo total (FT), alcalinidade $\mathrm{HCO}_{3}{ }^{-}$, cloreto, dureza, oxigênio consumido (OC), oxigênio dissolvido (OD), condutividade e demanda bioquímica de oxigênio $\left(\mathrm{DBO}_{5,20}\right)$ referentes às medições mensais realizadas entre o ano de 1998 a 2012, com exceção dos anos 2003 e 2004 não disponibilizados pela CESAMA, totalizando 13 anos de monitoramento.

Para a cidade de Juiz de Fora, o Plano Diretor de Desenvolvimento Urbano - PDDU (Juiz de Fora, 2000), estabelece que há uma sazonalidade bem definida no município, sendo a estação chuvosa de outubro a abril, com temperaturas mais elevadas e maiores precipitações (média de $20,9{ }^{\circ} \mathrm{C}$ e $199 \mathrm{~mm}$ por mês) e a estação seca de maio a setembro, período mais frio e com menor presença de chuvas (média de $17,1^{\circ} \mathrm{C}$ e $40 \mathrm{~mm}$ por mês).

Procedeu-se ao cálculo das médias de cada parâmetro para as duas estações anualmente e verificou-se a ocorrência de normalidade (Teste Kolmogorov-Smirnov). Foi aplicado o teste $\mathrm{t}$ de Student, com o auxílio do Microsoft Office Excel 2010, para verificar a ocorrência de variação estatisticamente significativa $(\mathrm{p}<0,05)$ entre as médias históricas das matrizes 
formadas com os dados de seca e chuva dos treze anos monitorados.

Alguns dados não foram mensurados durante vários meses de um mesmo ano, impossibilitando calcular uma média referente à estação daquele ano. Por esse motivo, o parâmetro ferro (Fe) foi excluído nos anos de 1998 e 1999; o manganês (Mn) e a condutividade foram excluídos de 1998 a 2002; os dados de fósforo total (FT) foram excluídos nos anos de 1998 a 2002 e 2005; os OD foram excluídos nos anos de 2000, 2002 e 2011; e a $\mathrm{DBO}_{5,20}$ nos anos de 1998 a 2002 e 2011.

Verificou-se a adequação dos dados à AF por meio dos testes de Kaiser-Meyer-Olkin (KMO) e esfericidade de Bartlett. As análises multivariadas (AF / ACP) foram realizadas no software SPSS ${ }^{\circledR}$ 15.0, e consistiram em três etapas, descritas por Toledo e Nicolella (2002): elaboração da matriz de correlação; extração dos fatores comuns com possível redução das variáveis explicativas e rotação dos eixos relativos aos fatores comuns, visando facilitar e simplificar a interpretação, somente quando necessário.

Os dados originais, em forma de matriz, foram expressos por $X=\left(X_{i, j}\right)$ em que, $i=1 \ldots n$ amostragens e $\mathrm{j}=1 \ldots \mathrm{p}$ variáveis limnológicas. As amostragens abrangeram os anos de $1998 \mathrm{a}$ 2012, com exceção dos anos 2003 e 2004, havendo, entretanto, ausência de dados em vários meses, os quais foram desconsiderados nas análises.

O coeficiente de correlação superior a 0,5 expressa uma forte correlação entre as variáveis limnológicas (Cohen, 1988 e Helena et al., 2000). Valores entre 0,3 e 0,49 expressam uma moderada correlação (Cohen, 1988). Para extração das componentes principais, utilizou-se a matriz de correlação com vista a eliminar o problema de escalas e unidades diferenciadas em que as variáveis foram medidas. A principal característica da $\mathrm{ACP}$, além da ortogonalidade, é que as componentes principais são obtidas em ordem decrescente de máxima variância. Assim, a primeira componente explica o máximo da variabilidade total dos dados; a segunda, explica o máximo da variabilidade dos dados restantes, não correlacionados com a primeira e assim sucessivamente (Guedes et al., 2012).

Os resultados da ACP, em alguns casos, podem apresentar dificuldades na sua interpretação. Objetivando superar tal obstáculo, adota-se o procedimento de rotação ortogonal da matriz das cargas fatoriais que possibilita melhor interpretação dos fatores ao redistribuir a variância explicada pelas componentes, não alterando na variância acumulada do conjunto de componentes. A utilização da rotação ortogonal pelo método Varimax permite um melhor ajuste ao modelo fatorial possível de explicação, sendo frequentemente utilizada em estudos de qualidade de água e processos hidrológicos (Aprile e Farias, 2001; Andrade et al., 2007; Girão et al., 2007; Guedes et al., 2012).

\section{RESULTADOS E DISCUSSÃO}

Os resultados das análises preliminares, que visaram testar a adequacidade dos dados à $\mathrm{AF} / \mathrm{ACP}$, possibilitaram o emprego de tal metodologia. Ao testar a hipótese de que a matriz de correlação é uma matriz identidade, teste de esfericidade de Bartlett $(\mathrm{p} \approx 0,000)$, foi descartada a hipótese nula, verificando a existência de correlações significativas entre as variáveis. Outro teste utilizado foi o índice de adequação da amostra (KMO), que segundo Hair et al. (2007), quantifica o grau de intercorrelação entre as variáveis. O resultado encontrado foi $\mathrm{KMO}=0,701$, o que permite a aplicação da análise.

Informações relevantes sobre a dinâmica das variáveis pode ser obtida através da matriz de correlação. Os parâmetros limnológicos que possuíram maior número de correlações (Tabela 1) tiveram maior importância na composição das componentes principais (CPs). A variável $\mathrm{pH}$ foi eliminada por apresentar correlações fracas com as outras variáveis e o teste foi refeito. No processamento da AF / ACP, a partir da matriz de correlação, foram extraídas as comunalidades, que representam o nível de associação entre a variável e os fatores 
extraídos, ou seja, a porcentagem da variância da variável explicada pelos fatores utilizados (Toledo e Nicolella, 2002). Valores abaixo de 0,5 indicam que a variável deve ser eliminada e a AF / ACP refeita (Figueiredo Filho e Silva Júnior, 2010). Norteados por tais considerações, eliminou-se a dureza, a alcalinidade e o manganês da análise.

Tabela 1. Matriz de correlação das variáveis limnológicas da Represa Dr. João Penido.

\begin{tabular}{lrrrrrrrr}
\hline & Cor & Turbidez & Fe & Ptotal & Cloreto & Condut. & DBO & OD \\
\hline Cor & 1,000 & & & & & & & \\
Turbidez & 0,470 & 1,000 & & & & & & \\
Fe & 0,736 & 0,324 & 1,000 & & & & & \\
Ptotal & $-0,208$ & $-0,124$ & $-0,049$ & 1,000 & & & & \\
Cloreto & 0,202 & 0,011 & 0,313 & 0,384 & 1,000 & & & \\
Condut. & 0,594 & 0,451 & 0,472 & $-0,102$ & 0,170 & 1,000 & & \\
DBO & 0,332 & $-0,177$ & 0,297 & $-0,225$ & 0,194 & 0,188 & 1,000 & \\
OD & 0,616 & 0,406 & 0,468 & $-0,133$ & 0,204 & 0,340 & 0,041 & 1,000 \\
\hline
\end{tabular}

As três primeiras componentes, explicaram cerca de $73 \%$ da variância total dos dados. Embora não exista um critério consensual sobre o número de fatores que devem ser extraídos na análise, o critério de Kaiser sugere a extração dos fatores com autovalor acima de um (Figueiredo Filho e Silva Júnior, 2010). Já Hair et al. (2007) sugerem que o número de componentes que representem $60 \%$ da variância acumulada, refletem uma ideia aceitável da variância original.

A Tabela 2 apresenta a matriz não rotacionada de pesos fatoriais que indica a contribuição que cada variável possui na componente principal. Esse valor deve ser maior que o valor absoluto de 0,5 e deve corresponder a uma única componente. O parâmetro OC apresentou valores maiores que 0,5 em mais de uma componente, mesmo após rotação. Optou-se por eliminá-lo e refazer o teste, resultando na Tabela 2. Caso semelhante aconteceu com a turbidez, porém, ao rotacionar a matriz pelo método Varimax, o problema foi superado conforme Tabela 3.

Tabela 2. Matriz de pesos fatoriais das variáveis limnológicas nas três componentes principais selecionadas.

\begin{tabular}{lccc}
\hline \multirow{2}{*}{ Variáveis } & \multicolumn{3}{c}{ Matriz(a) } \\
\cline { 2 - 4 } & \multicolumn{3}{c}{ Componente Principal (CP) } \\
\cline { 2 - 4 } & 0,915 & 2 & 3 \\
\hline Cor & 0,595 & $-0,058$ & $-0,080$ \\
Turbidez & 0,815 & $-0,291$ & 0,552 \\
Fe & $-0,196$ & 0,171 & $-0,108$ \\
Ptotal & 0,327 & 0,788 & 0,396 \\
Cloreto & 0,732 & 0,817 & $-0,016$ \\
Condutividade & 0,332 & $-0,044$ & 0,067 \\
DBO & 0,715 & 0,128 & $-0,858$ \\
OD & 3,16 & $-0,051$ & 0,211 \\
Autovalor & 39,45 & 1,43 & 1,27 \\
\% Variância Explicada & 17,82 & 15,81 \\
$\%$ Variância Acumulada & 39,45 & 57,27 & 73,09 \\
\hline
\end{tabular}

Nota: Os três tons de cinza destacam o peso de cada variável para aquela Componente Principal (CP). 
A primeira componente, após a aplicação do algoritmo Varimax (FV1), explicou cerca de $38,2 \%$ da variância total dos dados e teve como variável mais expressiva a cor, cujo valor foi de 0,879 (Tabela 3 ).

Tabela 3. Matriz de pesos fatoriais das três componentes principais após rotação Varimax.

\begin{tabular}{lccc}
\hline \multirow{2}{*}{ Variáveis } & \multicolumn{3}{c}{ Matriz(a) Rotacionada } \\
\cline { 2 - 4 } & \multicolumn{3}{c}{ Fator Varimax (FV) } \\
\cline { 2 - 4 } & 0,879 & $-0,029$ & 0,269 \\
\hline Cor & 0,722 & $-0,137$ & $-0,451$ \\
Turbidez & 0,754 & 0,184 & 0,320 \\
Fe & $-0,183$ & 0,844 & $-0,265$ \\
Ptotal & 0,238 & 0,809 & 0,251 \\
Cloreto & 0,731 & 0,007 & 0,091 \\
Condutividade & 0,132 & $-0,043$ & 0,918 \\
DBO & 0,745 & 0,030 & $-0,052$ \\
OD & 3,06 & 1,42 & 1,37 \\
Autovalor & 38,23 & 17,78 & 17,08 \\
\% Variância Explicada & 38,23 & 56,01 & 73,09 \\
\% Variância Acumulada & &
\end{tabular}

Nota: Os três tons de cinza destacam o peso de cada variável para o Fator Varimax - FV.

As outras variáveis desta componente também apresentaram resultados expressivos, cujos valores estão acima de 0,700 . Dentre as variáveis estudadas, a cor e o Fe foram as que apresentaram maior número de correlações, inclusive possuindo entre si, o maior coeficiente de Pearson da matriz (Tabela 1). Isso se justifica pelo fato do Fe, na ausência de oxigênio dissolvido, se apresentar em sua forma reduzida $\left(\mathrm{Fe}^{2+}\right)$, podendo se oxidar e precipitar causando coloração na água (Von Sperling, 2005). Os resultados obtidos pelo teste $t$ de Student destacam variação sazonal para a cor e o Fe com elevação de suas médias na estação chuvosa (Tabela 4), o que já foi observado por Rocha et al. (2014). Além disso, a turbidez está intrinsecamente relacionada com a cor presente na água, de forma que quando a cor aumenta, a turbidez também pode aumentar (Von Sperling, 2005). Para a CETESB (2009), a condutividade está relacionada com a presença de sais na água, indicando indiretamente uma medida da concentração de poluentes. A condutividade da água é proporcional ao aumento de sólidos dissolvidos, o que pode explicar a presença da condutividade nessa componente, visto que a cor também está relacionada com a presença dos sólidos dissolvidos. Uma fonte importante para o OD é a produção fotossintética (CETESB, 2009). Dessa forma, o OD se relaciona nessa componente, devido a presença de cor e turbidez que influenciam na passagem dos raios solares no corpo d'água e com isso podem diminuir a disponibilidade de luz para a fotossíntese e consequentemente diminuir a geração de OD na água.

A segunda componente (FV2) que explicou cerca de 17,8\% da variância dos dados foi composta pelos parâmetros fósforo total e cloreto. A presença de fósforo nas águas pode ter origem na dissolução de compostos do solo (escala muito pequena), despejos domésticos e/ou industriais, detergentes, excrementos de animais e fertilizantes. Concentrações elevadas de fósforo podem contribuir, da mesma forma que o nitrogênio, para a proliferação de algas e acelerar o processo de eutrofização. Assim como o fósforo total, a presença de cloreto também está relacionada ao lançamento de efluentes domésticos no corpo hídrico. Enquanto o fósforo é proveniente, principalmente de detergentes e excretas de animais, o cloreto está presente na urina humana e estima-se que cada pessoa expele através da urina cerca $6 \mathrm{~g}$ de 
cloreto por dia, o que faz com que os esgotos apresentem concentrações de cloreto que ultrapassam a $15 \mathrm{mg} . \mathrm{L}^{-1}$ (CETESB, 2009).

A componente FV3 foi representada por uma única variável, a DBO. Dentre todas as componentes, essa foi a mais expressiva, com valor de 0,918. Além disso, a variável explicou cerca de $17 \%$ da variância total dos dados. Esse resultado reforça a importância desse parâmetro, que é um dos mais utilizados para indicação da qualidade da água. A DBO (Demanda Bioquímica de Oxigênio) se refere a quantidade de oxigênio necessária para degradar a matéria orgânica presente no meio. Os maiores aumentos em termos de DBO, num corpo d'água, são provocados por despejos de origem predominantemente orgânica. A presença de um alto teor de matéria orgânica pode induzir ao completo esgotamento do oxigênio na água, provocando o desaparecimento de peixes e outras formas de vida aquática. Um elevado valor da DBO pode indicar um incremento da microflora presente e interferir no equilíbrio da vida aquática (CETESB, 2009).

Tabela 4. Resultados do Teste t-Student e médias e desvios padrões das variáveis monitoradas.

\begin{tabular}{|c|c|c|c|}
\hline \multirow{2}{*}{ Variável } & \multirow{2}{*}{ Teste T-Student } & \multicolumn{2}{|c|}{ Média e Desvio Padrão } \\
\hline & & Seca & Chuva \\
\hline $\mathrm{pH}$ & 0,082 & $7,037 \pm 0,216$ & $6,934 \pm 0,209$ \\
\hline Cor $(\mathrm{mg} \mathrm{PtCo} / \mathrm{L})$ & 0,000 & $38,654 \pm 13,009$ & $68,067 \pm 22,508$ \\
\hline Turbidez (UNT) & 0,001 & $4,825 \pm 0,990$ & $6,305 \pm 1,216$ \\
\hline $\mathrm{Fe}\left(\mathrm{mg} \cdot \mathrm{L}^{-1}\right)$ & 0,001 & $0,695 \pm 0,258$ & $1,718 \pm 0,802$ \\
\hline $\operatorname{Mn}\left(m g \cdot L^{-1}\right)$ & 0,932 & $0,207 \pm 0,200$ & $0,214 \pm 0,089$ \\
\hline P total $\left(\mu \mathrm{g} . \mathrm{L}^{-1}\right)$ & 0,941 & $0,346 \pm 0,181$ & $0,333 \pm 0,118$ \\
\hline Alcalinidade $\mathrm{CO} 3$ & 0,546 & $10,609 \pm 2,871$ & $11,164 \pm 2,730$ \\
\hline Cloreto (mg. $\left.\mathrm{L}^{-1}\right)$ & 0,372 & $9,100 \pm 5,098$ & $10,854 \pm 8,676$ \\
\hline Dureza $\left(m g . L^{-1}\right)$ & 0,633 & $11,782 \pm 5,877$ & $11,823 \pm 4,731$ \\
\hline OC $\left(m g \cdot L^{-1}\right)$ & 0,004 & $2,390 \pm 0,481$ & $3,038 \pm 0,747$ \\
\hline $\mathrm{OD}\left(\mathrm{mg} \cdot \mathrm{L}^{-1}\right)$ & 0,377 & $5,903 \pm 1,266$ & $6,327 \pm 0,835$ \\
\hline Condutividade (uS/cm) & 0,001 & $18,347 \pm 1,447$ & $21,134 \pm 0,874$ \\
\hline DBO (mg. $\left.\mathrm{L}^{-1}\right)$ & 0,408 & $2,728 \pm 3,015$ & $4,135 \pm 6,352$ \\
\hline
\end{tabular}

O comportamento das variáveis e sua distribuição pelas componentes permitem verificar as relações que estas apresentam entre si. Dessa forma, assim como feito por outros autores (Helena et al., 2000; Andrade et al., 2007; Guedes et al., 2012), é possível atribuir um nome que represente as características comuns entre tais variáveis de cada componente. Logo, a FV1 reflete a influência de sólidos dissolvidos associados ao escoamento superficial pelo fato das variáveis com os maiores coeficientes terem apresentado diferenças significativas entre as estações de seca e chuva. A FV2 se associa fortemente a possíveis lançamentos de esgoto na área da bacia. A concentração de fósforo total se mostrou muito elevada nas duas estações, pois a média para o período seco foi de $0,346 \mathrm{mg} . \mathrm{L}^{-1}$ e para o período chuvoso foi de 0,333 mg. $\mathrm{L}^{-1}$. Esses valores são cerca de 3 vezes maiores que o permitido pela Resolução CONAMA 357/2005 (Brasil, 2005) para águas doces de Classe $1\left(0,1 \mathrm{mg} . \mathrm{L}^{-1} \mathrm{P}\right)$. A FV3, assim como a FV2, caracteriza a influência do lançamento de esgotos na área da bacia, como já foi observado por Rocha et al. (2014).

Os resultados obtidos pelo teste t de Student apontaram as variáveis cor, turbidez, ferro, 
OC e condutividade com diferença sazonal significativa $(\mathrm{p}<0,05)$. Todas com aumento nas médias no período chuvoso. Contudo, não se descarta a hipótese de que outras variáveis também sofram interferência da precipitação, uma vez que é difícil mensurar temporalmente as influências das chuvas sobre as variáveis limnológicas (Fritzsons et al., 2003). Acrescenta-se o fato das amostras coletadas serem provenientes da captação, onde a qualidade da água pode ser resultado, dentre outros processos, do efeito de tamponamento ao longo do curso hídrico e da decantação na Represa, semelhante ao descrito por Carvalho et al. (2000) e Padial et al. (2009).

Observa-se também que o parâmetro $\mathrm{DBO}_{5,20}$ apresentou valores de desvio padrão, tanto para a estação seca quanto para a chuvosa, maiores que os valores das médias. Isso indica que os dados desse parâmetro estão variando muito. Esses altos valores de desvio padrão também poderiam ser explicados pela grande lacuna de dados desse parâmetro, entre 1998-2002 e 2011.

Ao se considerar que a componente FV1, a que mais explicou as variâncias totais dos dados, é resultante do transporte e lixiviação de materiais alóctones, deduz-se que o uso e a ocupação irregular do solo no entorno da sub-bacia influencia diretamente na qualidade da água deste manancial. Carneiro et al. (2010) afirmam que há uma desarticulação entre os instrumentos de gerenciamento dos recursos hídricos e os de planejamento do uso do solo, marcada pelo forte grau de informalidade e até mesmo ilegalidade na ocupação do solo. Este fato é nitidamente observado na sub-bacia de contribuição da Represa Dr. João Penido, sendo que resultados semelhantes foram encontrados por Rocha et al. (2014) num período de sete anos de monitoramento.

\section{CONCLUSÃO}

A utilização do teste $t$ de Student confirmou a necessidade de estudos que contemplem diferentes estações (seca e chuva), tendo em vista a variação observada para importantes parâmetros limnológicos em virtude da pluviosidade.

A Análise Fatorial / Análise de Componentes Principais promoveu a redução do número de variáveis de qualidade da água: dos treze parâmetros analisados, cinco foram eliminados, o que facilita a interpretação dos dados que mais influenciaram na dinâmica desta sub-bacia e diminuindo custos com monitoramento.

A análise permitiu a seleção de três componentes principais que explicaram $73,1 \%$ da variação total dos dados, indicando o escoamento superficial como principal determinante das variáveis que compõem a primeira componente e a contribuição orgânica, não associada à precipitação pluviométrica, como sugestiva das variáveis das segunda e terceira componentes.

A seleção de parâmetros de monitoramento de qualidade da água é relevante para o gerenciamento de recursos hídricos, consideradas as especificidades de cada sub-bacia e manancial correspondente. As informações obtidas podem apoiar decisões sobre planejamento e uso da terra, além de reduzir gastos com monitoramento.

\section{AGRADECIMENTOS}

Os autores agradecem a UFJF pelas bolsas concedidas e aos pesquisadores do NAGEA, LADINAA e PGECOL pelas discussões.

\section{REFERÊNCIAS}

AB'SÁBER, A. N. Do Código Florestal para o Código da Biodiversidade. Biota Neotrópica, v. 10, n. 4, p. 331-335, 2010. http://dx.doi.org/10.1590/S1676-06032010000400037 
ABILDTRUP, J.; GARCIA, S.; STENGER, A. The effect of forest land use on the cost of drinking water supply: A spatial econometric analysis. Ecological Economics, n. 92, p. 126-136, 2013. http://dx.doi.org/10.1016/j.ecolecon.2013.01.004

AKBAR, T. A.; HASSAN, Q. K.; ACHARI, G. A methodology for clustering lakes in Alberta on the basis of water quality parameters. Clean - Soil, Air, Water, v. 39, n. 10, p. 916-924, 2011. http://dx.doi.org/10.1002/clen.201100050

ANDRADE, E. M.; ARAÚJO, L. F. P.; ROSA, M. F.; DISNEY, W.; ALVES, A. B. Seleção dos indicadores da qualidade das águas superficiais pelo emprego da análise multivariada. Engenharia Agrícola, v. 27, n. 3, p. 683-690, 2007. http://dx.doi.org/10.1590/S0100-69162007000400011

APRILE, F. M.; FARIAS, V. P. Avaliação da qualidade da água da bacia do Rio Goiana, Pernambuco - Brasil. Bioikos, v. 15, n. 2, p. 109-114, 2001.

AUTIN, M. A.; EDWARDS, D. Nonparametric harmonic regression for estuarine water

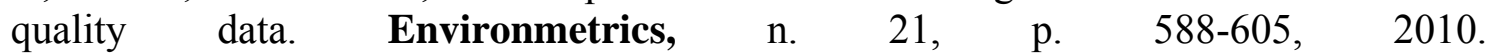
http://dx.doi.org/10.1002/env.1013

BABOROWSKI, M.; SIMEONOV, V.; EINAX, J. Assessment of water quality in the Elbe River at flood water conditions based on cluster analysis, principle components analysis, and source apportonment. Clean - Soil, Air, Water, v. 40, n. 4, p. 373-380, 2012. http://dx.doi.org/10.1002/clen.201100085

BERNARDI, J. V. E.; LACERDA, L. D.; DÓREA, J. G.; LANDIM, P. M. B.; GOMES, J. P. O.; ALMEIDA, R. et al. Aplicação da análise das componentes principais na ordenação dos parâmetros físico-químicos no alto Rio Madeira e afluentes, Amazônia Ocidental. Geochimica Brasiliensis, v. 23, n. 1, p. 79-90, 2009.

BHATTACHARYYA, K. G.; KAPIL, N. Impact of urbanization on the quality of water in a naturalreservoir: a case study with the DeeporBeel in Guwahaticity, India. Water and Environment Journal, n. 24, p. 83-96, 2010. http://dx.doi.org/10.1111/j.17476593.2008.00157.x

BRASIL. Lei n 12.651, de 25 de maio de 2012. Diário Oficial [da] União, Brasília, 28 mai. 2012a. Disponível em: http://www.planalto.gov.br/civil_03/_ Ato20112014/2012/Lei/L12651.htm. Acesso em: 08 out. 2013.

BRASIL. Lei $\mathrm{n}^{\circ}$ 12.727, de 17 de outubro de 2012. Diário Oficial [da] União, Brasília, 17 out. 2012b. Disponível em: http://www.planalto.gov.br/civil_03/_Ato20112014/2012/Lei/L12727.htm. Acesso em: 10 nov. 2013.

BRASIL. Resolução 357, de 17 de março de 2005. Diário Oficial [da] União, Brasília, 17 mar. 2005. Disponível em www.mma.gov.br/port/conama/res/res 05/res35705.pdf. Acesso em: 10 fev. 2014.

CARNEIRO, P. R. F.; CARDOSO, A. L.; ZAMPRONIO, G. B.; MARTINGIL, M. de C. A gestão integrada de recursos hídricos e do uso do solo em bacias urbano-metropolitanas: o controle de inundações na bacia dos rios Iguaçu/Sarapuí, na Baixada Fluminense. Ambiente e Sociedade, v. 13, n. 1, p. 29-49, 2010.

CARVAlHO, A. R.; SCHLITTLER, F. H. M.; TORNISIELO, V. L. Relações da atividade agropecuária com parâmetros físicos químicos da água. Química. Nova, v. 23, n. 5, p. 618-622, 2000. http://dx.doi.org/10.1590/S0100-40422000000500009 
CASATTI, L. Alterações no Código Florestal Brasileiro: impactos potenciais sobre a ictiofauna. Biota Neotrópica, v. 10, n. 4, p. 31-34, 2010. http://dx.doi.org/10.1590/S1676-06032010000400002

COMPANHIA DE SANEAMENTO MUNICIPAL DE JUIZ DE FORA - CESAMA. Represa Dr. João Penido. Disponível em: http://www.cesama.com.br/? pagina=joaopenido Acesso em: 29 dez. 2014.

COMPANHIA AMBIENTAL DO ESTADO DE SÃO PAULO - CETESB. Qualidade das águas interiores no estado de São Paulo. São Paulo, 2009. 43p.

COHEN, J. Statistical power analysis for the behavioral sciences. 2. ed. Hillsdale Lawrence Erlbaum Associates, 1988. 567p.

FIGUEIREDO FILHO, D. B.; SILVA JÚNIOR, J. A. da. Visão além do alcance: uma introdução à análise fatorial. Opinião Pública, v. 16, n. 1, p. 160-185, 2010. http://dx.doi.org/10.1590/S0104-62762010000100007

FRINTZONS, E.; HIND, E. C.; MANTOVANI, L. E.; RIZZI, N. E. As alterações da qualidade da água do Rio Capivari com o deflúvio: um instrumento de diagnóstico de qualidade ambiental. Engenharia Sanitária e Ambiental, v. 8, n. 4, p. 239-248, 2003.

GIRÃO, E. G.; ANDRADE, E. M.; ROSA, M. de F.; ARAÚJO, L. de F. P.; MEIRELES, A. C. M. Seleção dos indicadores da qualidade de água no Rio Jaibaras pelo emprego da análise de componente principal. Revista Ciência Agronômica, v. 38, n. 1, p. 17-24, 2007.

GUEDES, H. A. S.; SILVA, D. D.; EleSBON, A. A. A.; RIBEIRO, C. B. M.; MATOS, A. T.; SOARES, J. H. P. Aplicação da análise estatística multivariada no estudo da qualidade da água do Rio Pomba, MG. Revista Brasileira de Engenharia Agrícola e Ambiental, v. 16, n. 5, p. 558-563, 2012. http://dx.doi.org/10.1590/S141543662012000500012

HAIR, J. J. F.; ANDERSON, R. E.; TATHAM, R. L.; BLACK, W. C. Análise multivariada de dados. 5. ed. Porto Alegre: Bookman, 2007. 593p.

HELENA, B.; PARDO, R.; VEGA, M.; BARRADO, E.; FERNÁNDEZ, J. M.; FERNÁNDEZ, L. Temporal evolution of groundwater composition in an alluvial aquifer (Pisuerga River, Spain) by principal component analysis. Water Research, v. 34, n. 3, p. 807-816, 2000. http://dx.doi.org/10.1016/S0043-1354(99)00225-0

INSTITUTO BRASILEIRO DE GEOGRAFIA E ESTATÍSTICA - IBGE. Cidades@: MG Juiz de Fora. Disponível em: http://cod.ibge.gov.br/234B9. Acesso em: 07 maio 2014.

JUIZ DE FORA. Lei $\mathbf{n}^{\mathbf{0}} \mathbf{1 1 . 8 1 7}$ de 04 de agosto de 2009. Altera a Lei Municipal $\mathrm{n}^{\circ} 6.087$, de 04 de dezembro de 1981, e dá outras providências. Disponível em: http://www.leismunicipais.com.br/legislacao-de-juiz-de-fora/973791/lei-11817-2009juiz-de-fora-mg.html Acesso em: 07 jun. 2014

JUIZ DE FORA. Lei $\mathbf{n}^{\mathbf{0}} \mathbf{9 . 8 1 1}$ de 27 de junho de 2000. Institui o plano diretor de desenvolvimento urbano de Juiz de Fora. Disponível em: http://www.leismunicipais.com.br/legislacao-de-juiz-de-fora/1391368/lei-9811-2000juiz-de-fora-mg.html Acesso em 07 jun. 2014. 
JUIZ DE FORA. Lei $\mathbf{n}^{\mathbf{0}}$ 6.910 de 31 de maio de 1986. Dispõe sobre o ordenamento do uso e ocupação do solo no município de Juiz de Fora. Disponível em: http://www.leismunicipais.com.br/legislacao-de-juiz-de-fora/1060646/lei-6910-1986juiz-de-fora-mg.html. Acesso em: 02 jun. 2014.

JUIZ DE FORA. Lei $\mathbf{n}^{\circ} \mathbf{6 . 0 8 7}$ de 04 de dezembro de 1981. Dispõe sobre o parcelamento, uso e ocupação do solo, na área da bacia hidrográfica da Represa Dr. João Penido, em Juiz de Fora e dispõe outras providências. Disponível em: http://www.leismunicipais.com.br/legislacao-de-juiz-de-fora/1380937/lei-6087-1981juiz-de-fora-mg.html. Acesso em: 07 jun. 2014

LIAO, S. W.; GAU, H. S.; LAI, W. L.; CHEN, J. J.; LEE, C. G. Identification of pollution of Tapeng Lagoon from neighbouring rivers using multivariate statistical method. Journal of Environmental Management, v. 88, n. 2, p. 286-292, 2008. http://dx.doi.org/10.1016/j.jenvman.2007.02.010

MACHADO, P. J. O. Crescimento urbano e ocupação de mananciais: o caso de Juiz de Fora/MG. In: FÓRUM AMBIENTAL DA ALTA PAULISTA, 2., 2006, Estância Turística de Tupã. Resumos... Estância Turística de Tupã: ANAP/FACCAT/UNESP, 2006. 1 CD-ROM.

MENDONÇA, E. C. C. N.; SOUZA, P. S. de. Aplicação da técnica de análise de componentes principais para caracterização de águas de poços artesianos de áreas urbanas de Goiânia e Aparecida de Goiânia. Plurais, v. 1, n. 1, p. 19-36, 2011.

PADIAL, P. R.; POMPÉO, M.; MOSCHINI-CARLOS, V. Heterogeneidade espacial e temporal da qualidade da água no reservatório Rio das Pedras (Complexo Billings, São Paulo). Revista Ambiente \& Água, v. 4, n. 3, p. 35-53, 2009. http://dx.doi.org/10.4136/1980-993X.

ROCHA, C. H. B.; COSTA, H. F. Variação temporal de parâmetros limnológicos em manancial de abastecimento em Juiz de Fora, MG. Revista Brasileira de Recursos Hídricos, v. 20, n. 2, p. 543-550, 2015.

ROCHA, C. H. B.; FREITAS, F. A.; SILVA, T. M. Alterações em variáveis limnológicas de manancial de Juiz de Fora devido ao uso da terra. Revista Brasileira de Engenharia Agrícola e Ambiental, v. 18, n. 4, p. 431-436, 2014. http://dx.doi.org/10.1590/S141543662014000400011

SHEELA, A. M.; LETHA, J.; JOSEPH, S.; CHACKO, M.; SANAL KUMAR, S. P.; THOMAS, J. Water quality assessment of a tropical coastal lake system using multivariate cluster, principal component and factor analysis. Lakes e Reservoirs: Research and Management, n. 17, p. 143-159, 2012. http://dx.doi.org/10.1111/j.14401770.2012.00506.x

SPEZIA, L. FUTTER, M. N. BREWER, J. Periodic multivariate Normal hidden Markov models for the analysis of water quality time series. Environmetrics, n. 22, p. 304-317, 2011. http://dx.doi.org/10.1002/env.1051

THURSTON, L. P. SMITH, C. A. GENSKOW, K. PROKOPY, L. S. HARGROVE, W. L. The social context of water quality improvement evaluation. New Directions for Evaluation, n. 135, p. 41-58, 2012. http://dx.doi.org/10.1002/ev.20026 
TOLEDO, L. G. de; NICOLELLA, G. Índice de qualidade de água em microbacia sob uso agrícola e urbano. Scientia Agrícola, v. 59, n. 1, p. 181-186, 2002. http://dx.doi.org/10.1590/S0103-90162002000100026

TU, J. Spatially varying relationships between land use and water quality across an urbanization gradient explored by geographically weighted regression. Applied Geography, n. 31, p. 376-392, 2011. http://dx.doi.org/10.1016/j.apgeog.2010.08.001

TUNDISI, J. G.; TUNDISI, T. M. Impactos potenciais das alterações do Código Florestal nos recursos hídricos. Biota Neotrópica, v. 10, n. 4, p. 67-75, 2010. http://dx.doi.org/10.1590/S1676-06032010000400010

VANZELA, L. S.; HERNADEZ, F. B. T.; FRANCO, R. A. M. Influência do uso e ocupação do solo nos recursos hídricos do Córrego Três Barras, Marinópolis. Revista brasileira de Engenharia Agrícola e Ambiental, v. 14, n. 1, p. 55-64, 2010. http://dx.doi.org/10.1590/S1415-43662010000100008

VON SPERLING, M. Introdução à qualidade das águas e ao tratamento de esgotos. 3. ed. Belo Horizonte: Imprensa Universitária da UFMF, 2005. 452 p.

YU, D.; SHI, P.; LIU, Y.; XUN, B. Detecting land-use water quality relationships from the viewpoint of ecological restoration in an urban area. Ecological Engineering, n. 53, p. 205-216, 2013. http://dx.doi.org/10.1016/j.ecoleng.2012.12.045 